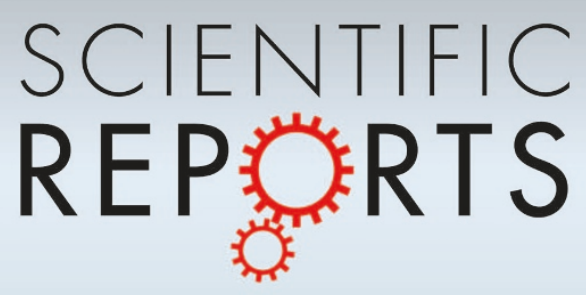

OPEN

SUBJECT AREAS:

BEHAVIOURAL ECOLOGY

EVOLUTIONARY ECOLOGY

Received

4 February 2014

Accepted

6 May 2014

Published

29 May 2014

Correspondence and requests for materials should be addressed to

I.-M.T. (spider@thu. edu.tw)

* Current address:

Evolution \& Ecology

Research Centre,

School of Biological,

Earth \& Environmental

Sciences, The

University of New

South Wales, Sydney

2052, Australia.

\title{
Evidence of bird dropping
} masquerading by a spider to avoid predators

\author{
Min-Hui Liv'1,3, Sean J. Blamires²*, Chen-Pan Liao² \& I. -Min Tso 1,2
}

\begin{abstract}
'Department of Life Science, National Chung-Hsin University, Taichung 40227, Taiwan, ${ }^{2}$ Department of Life Science, Tunghai University, Taichung 40704, Taiwan, ${ }^{3}$ Taiwan Endemic Species Research Institute, Nantou 55244, Taiwan.
\end{abstract}

Masquerading comes at various costs and benefits. The principal benefit being the avoidance of predators. The orb-web spider Cyclosa ginnaga has a silver body and adds a white discoid-shaped silk decoration to its web. The size, shape and colour of $C$. ginnaga's body resemble, when viewed by the human eye against its decoration, a bird dropping. We therefore hypothesized that their body colouration might combine with its web decoration to form a bird dropping masquerade to protect it from predators. We measured the spectral reflectance of: (i) the spider's body, (ii) the web decoration, and (iii) bird droppings, in the field against a natural background and found that the colour of the spider bodies and decorations were indistinguishable from each other and from bird droppings when viewed by hymentopteran predators. We monitored the predatory attacks on $C$. ginnaga when the spider's body and/or its decorations were blackened and found that predator attack probabilities were greater when only the decorations were blackened. Accordingly, we concluded that $C$. ginnaga's decoration and body colouration forms a bird dropping masquerade, which reduces its probability of predation.

nimal signals must be meaningful to an intended receiver to be effective ${ }^{1}$. There is, nevertheless, an
inevitable risk that unintended receivers, such as predators, may exploit these signals ${ }^{1,2}$. Masquerading,
crypsis and Batesian mimicry are examples of colourful body signals that may be expressed under high predation risk ${ }^{1,3}$.

Masquerading is when an animal's body colour and shape mimic an inanimate object. Crypsis is when an animal's body colouration resembles its background or obscures the position of the animal. Batesian mimicry is when an animal resembles an undesirable or unpalatable species in the eyes of its predator ${ }^{1,3,4}$. There is evidence from studies of avian predators searching for twig-mimicking caterpillars ${ }^{5,6}$ that the relative size of models and mimics affects the benefits of Batesian mimicry and masquerading, as does the context in which the mimics are found and their microhabitat selection.

The body colours of many web-building spiders are expressed at a compromise between being attractive to prey and inconspicuous to predators ${ }^{7-10}$. Some spider body colours, nevertheless, function to conceal the spider by crypsis $^{9,11,12}$, or they may masquerade as inanimate objects ${ }^{9,12,13}$. In addition to colourful bodies, many diurnal web-building spiders add conspicuously coloured decorations (also called stabilimenta) to their webs, which may be made from prey carcasses, egg sacs, plant detritus, or silk ${ }^{9,14}$. The decorations may, depending on their colouration and shape, function to attract prey ${ }^{9,14-17}$ or deter predators ${ }^{9,14,18-21}$. Like other components of spider webs, their expression inevitably comes with various costs and benefits ${ }^{15,18,22,23}$.

The conspicuously white silken, discoid-shaped, web decorations made by juveniles of the orb-web spider Cyclosa ginnaga function to attract prey but they may also attract predatory wasps ${ }^{21,24}$. Their silver bodies are conspicuous, to the eye, against their white decorations and, consequently, they are assumed to attract predators; inasmuch as predator attraction has been shown in other spiders with bright body colours ${ }^{25}$. The shape, size and colour of C. ginnaga's body against its white web decoration resemble, at least to a human observer, a typical bird dropping (Figure 1, also see Supplementary Figure S1 for additional examples). Bird dropping masquerading, accordingly, seems to be a reasonable hypothesis derived from human assessment. This hypothesis, nevertheless, has never been experimentally investigated.

Here we investigated whether the body colouration and web decorations of C. ginnaga might form a bird dropping masquerade using a combination of colourimetry and experimentation on wild spider subjects. We assumed support for the bird dropping masquerade hypothesis if: (1) the presence of the spider's decorations 

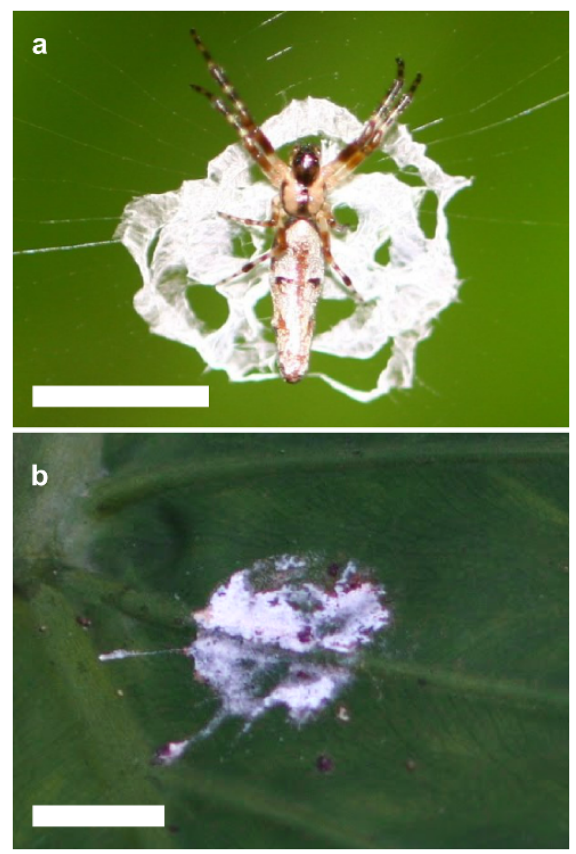

Figure $1 \mid$ Photograph of a juvenile $C$. ginnaga against its decoration (a) and a bird dropping (b) found at our study site (Scale bar: $5 \mathrm{~mm}$ ).

renders its body indistinguishable from bird droppings in the eyes of predators, and (2) concealing C. ginnaga's web decorations renders the spider conspicuous to predators, which we expect to be corroborated if we were to find a greater probability of predation on spiders on blackened decorations than on spiders on natural decorations.

\section{Results}

We measured the surface area of 125 C. ginnaga web decorations and 27 bird droppings and found no statistically significant difference (mean \pm SE: decorations $=0.63 \pm 0.065 \mathrm{~cm}^{2}$, droppings $=0.80 \pm$ $\left.0.122 \mathrm{~cm}^{2} ; t_{34}=-1.241 ; \mathrm{P}=0.223\right)$. Hence, $C$. ginnaga's web decorations resemble bird droppings in size.

The colour contrasts of the spider bodies when viewed by hymentopterans against its web decorations were significantly below 0.1 hexagon units; the colour discrimination threshold for unconditioned hymenopterans $\left(t_{9}=-18.12, P<0.0001\right)$ (Figure 2). Hence, as predicted under the bird dropping masquerading hypothesis, the body of $C$. ginnaga is indiscernible to wasp predators when viewed against its web decorations. The colour contrasts of the bird droppings when viewed against the spider's body $\left(t_{9}=-18.57, P<\right.$ $0.0001)$ or decorations $\left(t_{9}=-4.082, P<0.01\right)$ were also significantly below the hymenopteran colour discrimination threshold (Figure 2), likewise concurring with the bird dropping masquerading hypothesis.

The colour contrasts of the blackened spider bodies when viewed by hymenopterans against silk decorations that were covered with black powder (Figure 3 ) were significantly below the hymenopteran colour discrimination threshold $\left(t_{9}=-55.4387, P<0.0001\right)$. The colour contrast values of the decorations against the ink used to blacken the spider's body $\left(t_{7}=118.569, P<0.0001\right)$ and the spider bodies against the carbon powder used to blacken the decorations $\left(t_{9}=5.868, P=0.002\right)$ (Figure 3 ) were both significantly greater than the colour discrimination threshold for hymenopterans. Therefore, unmanipulated spiders would have been conspicuous to wasp predators when viewed against the blackened decorations. Likewise, the unmanipulated decorations would have been conspicuous to wasps when viewed against blackened spiders.

We placed video cameras perpendicular to C. ginnaga webs in the field and recorded 197 hours, 132 hours, 122 hours and 119 hours
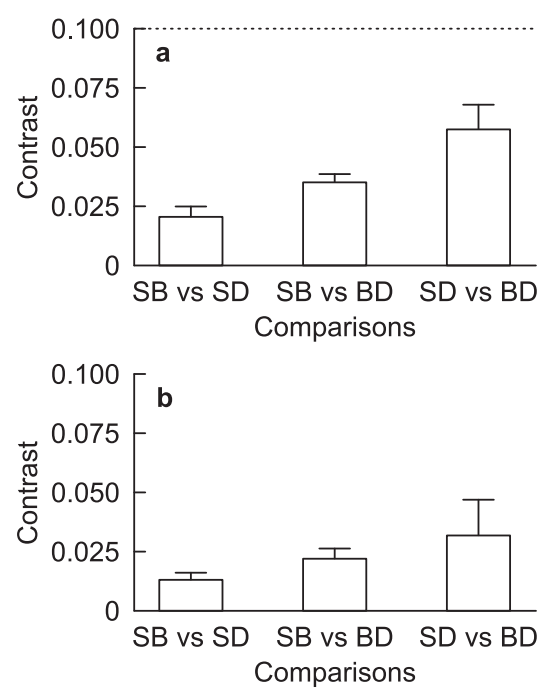

Figure $2 \mid$ Mean ( \pm SE) (a) chromatic and (b) achromatic contrast values. For spider body (SB), web decoration (SD) and bird dropping (BD) when viewed by hymenopterans against various backgrounds. The dashed line at 0.1 hexagon units represents the colour discrimination threshold for hymenopterans.

of footage for the "bodies and decorations exposed", "bodies concealed", "decorations concealed" and "bodies and decorations concealed" treatments respectively. We found that the wasp attack probabilities on unmanipulated spiders that only had their decorations blackened were significantly greater than that for any of the other groups (Table 1; Figure 4). When combined, the spider's body and its decoration are indistinguishable from bird droppings in the eyes of wasp predators. We found that: (1) the presence of the spider's decorations rendered its body indistinguishable from bird droppings in the eyes of wasp predators, (2) blackening C. ginnaga's web decorations rendered the spider conspicuous to wasps, and (3) spiders on blackened decorations had greater predation probabilities compared to spiders on natural decorations. These results lend
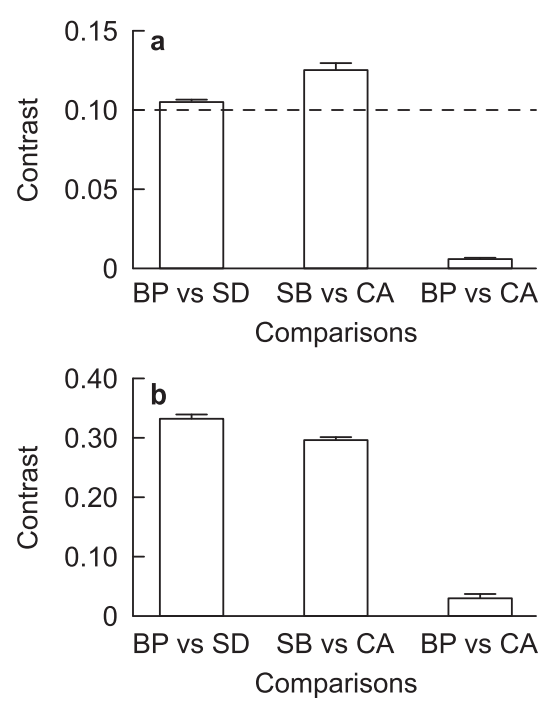

Figure $3 \mid$ Mean ( \pm SE) (a) chromatic and (b) achromatic contrast values. For spider body (SB), web decoration (SD), ink of the black pen (BP) and black carbon powder (CA) when viewed against different backgrounds. The dashed line represents 0.1 hexagon units; the color discrimination threshold for hymenopterans. 
Table 1 | The results of logistic regression comparing predator attack odds (probability of attack event/probability of non-attack event) across the four treatment groups, where the colour signals of decorations and/or spider were manipulated

\begin{tabular}{lccccc} 
Treatment & df & Estimate of $\beta$ & SE & Z & $P$ \\
\hline Intercept & 1 & -2.485 & 0.736 & -3.376 & 0.0007 \\
Control & 0 & 0 & 0 & - & - \\
Spider blackened & 1 & -0.348 & 1.265 & -0.275 & 0.7831 \\
Decoration blackened & 1 & 1.974 & 0.899 & 2.196 & 0.0281 \\
Both blackened & 1 & -0.080 & 1.272 & -0.063 & 0.9498
\end{tabular}

'The $\beta$ of the control group was arbitrarily designated as 0 to facilitate comparison of probabilities of different events.

${ }^{2}$ The ratio between probabilities of two certain events was $e^{\beta}$.

support to the hypothesis that the body and decorations of C. ginnaga form a bird dropping masquerade to avoid predators.

\section{Discussion}

Animal colour signals can be exploited by unintended receivers ${ }^{1-4,15}$. When the unintended receiver of a signal is a predator the consequences of exploitation are fatal, so selection pressure to offset such unintended attraction is strong ${ }^{1,26}$. Indeed, it is under such intensive selection pressure that strategies such as Batesian mimicry and masquerading are thought to evolve $e^{3-5}$. Here we showed that the bodies of juvenile C. ginnaga are indiscernible to predators when viewed against their white discoid-shaped web decorations. Moreover, the colour of C. ginnaga's body and decorations were indistinguishable from bird droppings in the eyes of their predators. Our results, therefore, strongly suggest that the body and web decorations of $C$. ginnaga combine to protect it from predators by forming a bird dropping masquerade.

To confidently ascribe masquerading it must nevertheless be demonstrated that predators repeatedly mistaken C. ginnaga's body and decorations as bird droppings ${ }^{4,5}$. Further observations of wasp responses to bird dropping are, therefore, required before masquerading can be definitively concluded. Furthermore, viewing orientation, distance and the viewing platform may affect the way masquerading prey are perceived by a predators ${ }^{27}$. The majority (i.e. 22 out of 27) of bird droppings recorded in our study site were orientated vertically on leaves, i.e. similar to the orientation of $C$. ginnaga on their webs, so we expected the vertical orientation of $C$. ginnaga to facilitate masquerade. We, nonetheless, suggest further investigations be done to assess how masqueraders suspended off a substrate, for example on a spider web, are viewed by hymenopteran predators compared to masqueraders on the surface of a substrate to be confident that the spiders resembled bird droppings in the eyes of wasps.

Many functions, including prey attraction, predator avoidance and web advertising (see Thery \& Casas $^{9}$, and Herberstein et al. ${ }^{14}$ for reviews), have been proposed for web decorations in different web-building spiders, including members of the genus Cyclosa $a^{18,19,28-30}$. This study, however, is the first to our knowledge to suggest that decorations might be used to form part of a bird dropping masquerade to avoid predators. Nevertheless, we concede that alternative explanations for the defensive influence of the spider body and decorations need to be examined to more thoroughly test the hypothesis that C. ginnaga's body colouration and web decorations form a bird dropping masquerade. For instance, further examinations are required to ascertain whether the decoration size or shape deters predators or obscures the spider's position, which may be determined by observations of wasps moving within the vicinity of the spider then moving away without launching an attack.

In summary, we used colourimetric measurements and performed field experiments to test the hypothesis that the body colouration and

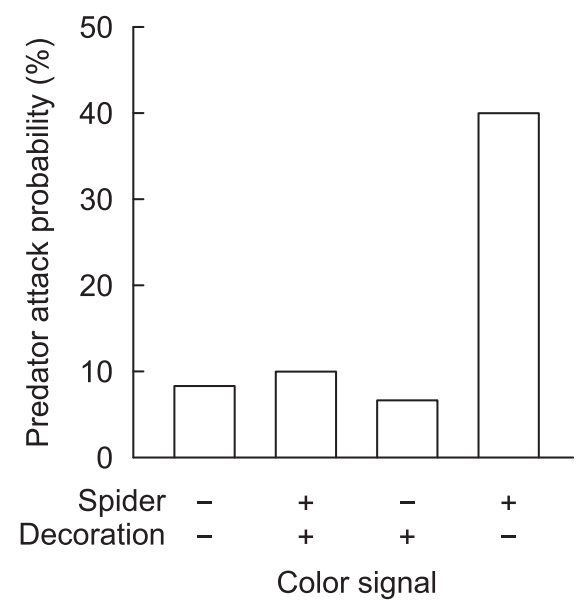

Figure $4 \mid$ Mean $( \pm S E)$ predator attack rates across our four treatments. "+" indicates treatments where the spider or decoration was exposed and "_" indicates treatments where the spider or decoration was blackened with a pen or carbon powder respectively.

web decorations of juvenile C. ginnaga interact to form a bird dropping masquerade and conceal them from predators. We found that: (1) the presence of the spider's decorations rendered its body indistinguishable from bird droppings in the eyes of its predators, and (2) concealing C. ginnaga's web decorations resulted in an increase in predatory attack probability. Accordingly, we concluded that the body and web decorations of C. ginnaga form a bird dropping masquerade to avoid predators. We, however, suggest that this conclusion be further tested against alternative explanations.

\section{Methods}

Study site and spiders. Cyclosa ginnaga is a relatively small (adults $<6 \mathrm{~mm}$ body length) orb-web spider that constructs webs in tropical forest understory throughout East Asia $^{31}$. As a juvenile it constructs a discoid-shaped white silk decoration around the hub of its web, where it positions itself with its forelegs evenly spread.

The following procedures were conducted over 13 days in September 2009 along a $300 \mathrm{~m}$ transect in a forest at Wu-Shy-Keng, Taichung, Taiwan (N24 $16^{\prime} 25.15^{\prime \prime}$, $\left.\mathrm{E} 120^{\circ} 56^{\prime} 53.51^{\prime \prime}\right)$.

Quantification of spider body and decoration colouration versus bird dropping coloration. We collected $10 \mathrm{C}$. ginnaga and measured the reflectance functions of their dorsal opithsoma relative to that of a tropical forest understory (i.e. a composite of shrubs and leaf litter) background across the 300-700 $\mathrm{nm}$ waveband range using a USB4000 spectrometer and OOIBase32 software (Ocean Optics, Dunedin, Florida, USA). We also collected and measured the reflectance functions of: (i) samples of decoration silk ( $n=8$ samples, collected and pooled from 40 different webs), (ii) six randomly encountered bird droppings (species unknown) found in the same site that the experiments were performed, as well as (iii) the black ink from the pen used to blacken C. ginnaga's body $(n=6)$ and (iv) the carbon powder used to blacken the decorations $(n=6)$ (Supplementary Figure S2).

We determined how wasps, the major predator observed in this study, viewed the decorations using a visual model derived from measurements of honeybee photoreceptor sensitivities ${ }^{32}$. We considered a honeybee vision model to be applicable to wasp predators because bees and wasps have similar types of photosensitive cells with similar pigments and sensitivities, occur in similar environments, and are phylogenetically closely related ${ }^{33,34}$.

We used Avicol (version 5.0) ${ }^{35}$ software to perform the following calculations. To calculate the chromatic and achromatic contrast values of bee photoreceptors when viewing: (i) the spider bodies, (ii) the decorations, (iii) bird droppings, (iv) the black ink or $(\mathrm{v})$ the carbon powder, we calculated the excitation values $\left(\mathrm{E}_{i}\right)$ of bee $\mathrm{UV}$, blue and green photoreceptors using the equations ${ }^{32}$ :

$$
Q_{i}=\int_{300}^{700} S(\lambda) D(\lambda) I_{S}(\lambda) d \lambda
$$

and

$$
\mathrm{E}_{i}=Q_{i} /\left(Q_{i}+1\right)
$$

Where: $Q_{i}$ is the quantum catch of the receptor under investigation $(i), S(\lambda)$ is the sensitivity function of the receptor under investigation, $D(\lambda)$ is the CIE standard daytime illumination function (D65) and $I_{s}(\lambda)$ is the signal (spider body, decorations, 
ink or powder, or forest understory background) reflectance function. The reflectance function of the forest understory background used was that previously measured at a similar study site ${ }^{25}$.

The excitation values of bee UV, blue and green photoreceptors were plotted as three-dimensional vectors onto a honeybee colour vision hexagon ${ }^{32}$. We calculated the chromatic contrast values as the Euclidean distance between signal (spider body, decorations, bird droppings, ink or powder) and background $\mathrm{E}_{i}$ values summed across photoreceptors in perceptual colour space. We calculated the achromatic contrasts as the excitation values of the green bee photoreceptors when viewing either spider bodies, decorations, bird droppings, ink or powder divided by those when viewing the background ${ }^{34}$.

Manipulation of spider body colouration and decorations and video processing. On each day of the study we collected 12 C. ginnaga directly from their webs and measured their body length and web decoration surface area $\left(=\pi \times\right.$ radius $\left.^{2}\right)$ using calipers to be sure that the spiders used for the procedures below had approximately similar body lengths and decoration surface areas. To ascertain whether surface area of C. ginnaga web decorations differed from nearby bird droppings we also measured the surface area of 27 randomly encountered bird droppings using calipers.

The spiders were randomly assigned to one of four treatment groups $[n=39$ (3 per day $\times 13$ days) per group] before being returned to their webs. The body colouration and web decorations of spiders in the first group were not altered. The bodies of spiders in the second group were concealed using a black marking pen. We did not conceal the bodies of spiders in the third group, but we concealed their web decorations using black carbon powder (Gestetuer MP4500, Ricoh, Japan). We concealed both the spider bodies and the web decorations of the fourth group. We coloured or powdered nearby vegetation using the same amount of ink or powder as used to cover the spider bodies or decorations to exclude the possibility that olfactory cues emitted from the ink or powder used confounded the experiment.

We placed video cameras (Sony HR118 Hi-8 DCR-TRV and DCR-SR series, Tokyo, Japan) perpendicular to each web at a distance of $\sim 1 \mathrm{~m}$. To mitigate potential confounding factors such as temporal changes in predator or prey abundance on the experiment, we simultaneously recorded three webs for each treatment (thus running 12 cameras at a time $)$ each day for $10 \mathrm{~h}(0730 \mathrm{~h}-1730 \mathrm{~h})$. We stopped recording only in the event of inclement weather. Data for spiders that occupied their web for $<4 \mathrm{~h}$ were not used in the analyses.

When the field experiments were completed we viewed the video footage in the laboratory at Tunghai University, Taichung, Taiwan. We defined predator attacks as either a predator (all wasps) moving toward the spider and launching an attack. Occasional camera positional adjustments, battery failure or other technical issues resulted in unequal hours of footage for each treatment, so we measured predator attack probabilities as the number of predator attacks observed per hour of footage. All footage was watched at slow speed by the same person (MHL) and every predator attack event was watched repeatedly, double-checking that all predator attacks were positively identified.

Analyses. We compared, using one tailed $t$-tests, the color contrasts of the bird droppings when viewed against spider bodies and web decorations with a honeybee chromatic discrimination threshold of 0.1 hexagon units. Although there are reports of honeybee color discrimination thresholds as low as 0.04 hexagon units for differentially conditioned bees ${ }^{36}$, we considered 0.1 to be more appropriate because it accounts for the different sensitivities of the UV, blue and green photoreceptors of absolute conditioned or unconditioned bees and wasps ${ }^{36,37}$. We also compared exposed and blackened spider bodies when viewed against the exposed and blackened decorations with the honeybee discrimination threshold. The predator attack data fitted a binomial distribution so we used a logistic regression to compare the predator attack probabilities of the four treatments. We performed all analyses using the program R (version 2.13.1).

1. Ruxton, G. D., Sherratt, T. N. \& Speed, M. P. Avoiding Attack, the Evolutionary Ecology of Crypsis, Warning Signals and Mimicry. (Oxford, U.K.: Oxford University Press, 2004)

2. Mougeot, F. \& Bretagnolle, V. Predation as a cost of sexual communication in nocturnal seabirds: an experimental approach using acoustic signals. Anim. Behav. 60, 647-656 (2000).

3. Skelhorn, J., Rowland, H. M., Speed, M. P. \& Ruxton, G. D. Masquerade: camouflage without crypsis. Science 327, 51 (2010).

4. Skelhorn, J., Rowland, H. M. \& Ruxton, G. D. The evolution and ecology of masquerade. Biol. J. Linn. Soc. 99, 1-8 (2010).

5. Skelhorn, J., Rowland, H. M., Delf, J., Speed, M. P. \& Ruxton, G. D. Densitydependent predation influences the evolution and behavior of masquerading prey. Proc. Nat. Acad. Sci. USA 108, 6532-6536 (2011).

6. Skelhorn, J. \& Ruxton, G. D. Size-dependent microhabitat selection by masquerading prey. Behav. Ecol. 24, 89-97 (2013).

7. Chuang, C. Y., Yang, E. C. \& Tso, I. M. Diurnal and nocturnal prey luring of a colorful predator. J. Exp. Biol. 210, 3830-3837 (2007).

8. Fan, C. M., Yang, E. C. \& Tso, I. M. Hunting efficiency and predation risk shapes the color-associated foraging traits of a predator. Behav. Ecol. 20, 808-816 (2009).

9. Théry, M. \& Casas, J. The multiple disguises of spiders: web colour and decorations, body colour and movement. Phil. Trans. Roy. Soc. B 364, 471-480 (2009).
10. Blamires, S. J. et al. Body spot coloration of a nocturnal sit-and-wait predator visually lures prey. Behav. Ecol. 23, 69-74 (2012).

11. Heiling, A. M., Herberstein, M. E. \& Chittka, L. Pollinator attraction - crab spiders manipulate flower signals. Nature 421, 334 (2003).

12. Théry, M. \& Casas, J. Predator and prey views of spider camouflage. Nature, 415, 133 (2002).

13. Eberhard, W. G. The natural history and behaviour of the bolas spider Mostphora dizzideani sp. N. (Araneidae). Psyche 87, 143-169 (1980).

14. Herberstein, M. E., Craig, C. L., Coddington, J. A. \& Elgar, M. A. The functional significance of silk decorations of orb-web spiders: a critical review of the empirical evidence. Biol. Rev. 75, 649-669 (2000).

15. Bruce, M. J., Herberstein, M. E. \& Elgar, M. A. Signalling conflict between prey and predator attraction. J. Evol. Biol. 14, 786-794 (2001).

16. Cheng, R. C. \& Tso, I. M. Signaling by decorating webs: luring prey or deterring predators? Behav. Ecol. 18, 1085-1091 (2007)

17. Blamires, S. J., Hochuli, D. F. \& Thompson, M. B. Why cross the web: decoration spectral properties and prey capture in an orb spider (Argiope keyserlingi) web. Biol. J. Linn. Soc. 94, 221-229 (2008).

18. Li, D. Q., Lim, M. L. M., Seah, W. K. \& Tay, S. L. Prey attraction as a possible function of discoid stabilimenta of juvenile orb-spinning spiders. Anim. Behav. 68, 629-635 (2004).

19. Chou, I. C., Wang, P. H., Shen, P. S. \& Tso, I. M. A test of prey-attracting and predator defence functions of prey carcass decorations built by Cyclosa spiders Anim. Behav. 69, 1055-1061 (2005)

20. Nakata, K. To be or not to be conspicuous: the effects of prey availability and predator risk on spider's web decoration building. Anim. Behav. 78, 1255-1260 (2009).

21. Tan, E. J. \& Li, D. Q. Detritus decorations of an orb-weaving spider, Cyclosa mulmeinensis (Thorell): for food or camouflage? J. Exp. Biol. 212, 1832-1839 (2009).

22. Bruce, M. J., Heiling, A. M. \& Herberstein, M. E. Spider signals: are web decorations visible to birds and bees? Biol. Lett. 1, 299-302 (2005).

23. Tseng, H. J., Cheng, R. C., Wu, S. H., Blamires, S. J. \& Tso, I. M. Trap barricading and decorating by a well-armored predator: extra protection or prey attraction? Behav. Ecol. Sociobiol. 65, 2351-2359 (2011).

24. Tan, E. J. et al. Why do orb-weaving spiders (Cyclosa ginnaga) decorate their webs with silk spirals and plant detritus? Anim. Behav. 79, 179-186 (2010).

25. Tso, I. M., Lin, C. W. \& Yang, E. C. Colourful orb-weaving spider, Nephila pilipes, through a bee's eyes. J. Exp. Biol. 207, 2631-2637 (2004).

26. Zuk, M. \& Kolluru, G. R. Exploitation of sexual signals by predators and parasitoids. Quar. Rev. Biol. 73, 415-438 (1998).

27. Skelhorn, J. \& Ruxton, G. D. Viewing distance affects how the presence of inedible models influence the benefit of masquerade. Evol. Ecol. 28, 441-455 (2014).

28. Tseng, L. \& Tso, I. M. A risky defence by a spider using conspicuous decoys resembling itself in appearance. Anim. Behav. 78, 425-431 (2009).

29. Blamires, S. J. et al. Multiple structures interactively influence prey capture efficiency in spider orb webs. Anim. Behav. 80, 947-953 (2010).

30. Gonzaga, M. O. \& Vasconcellos-Neto, J. Variation in the stabilimenta of Cyclosa fililineata Hingston, 1932, and Cyclosa morretes Levi, 1999 (Araneae: Araneidae), in southeastern Brazil. Psyche 2012, 396594 (2012).

31. Tanikawa, A. A revisional study of the Japanese spiders of the genus Cyclosa Menge (Araneae: Araneidae). Acta Arachnol. 41, 11-85 (1992).

32. Chittka, L. The colour hexagon: a chromaticity diagram based on photoreceptor excitation as a generalized representation of colour opponency. J. Comp. Physiol. A, 170, 533-543 (1992)

33. Peitsch, D. et al. The spectral input systems of hymenopteran insects and their receptor-based colour vision. J. Comp. Physiol. A 170, 23-40 (1992).

34. Osorio, D. \& Vorobyev, M. Photoreceptor sectral sensitivities in terrestrial animals: adaptations for luminance and colour vision. Proc. Roy. Soc. B 272, 1745-1752 (2005).

35. Gomez, D. AVICOL, A Program to Analyse Spectrometric Data. Available at http:// sites.google.com/site/avicolprogram/ (2006). Date of access 25/03/2014.

36. Dyer, A. G. \& Chittka, L. Fine colour discrimination requires differential conditioning in bumblebees. Naturwissenschaften 91, 224-227 (2004).

37. Dyer, A. G., et al. Parallel evolution of angiosperm colour signals: common evolutionary pressures linked to hymenopteran vision. Proc. Roy. Soc. B 279 , 3606-3615 (2012).

\section{Acknowledgments}

The work was supported by National Science Council, Taiwan (NSC-99-2632-B-029-001-MY3, NSC-102-2311-B-029-001-MY3) and Tunghai University Global Research \& Education on Environment and Society (GREEnS) project grants to I.M.T and a NSC postdoctoral grant (NSC-102-2811-B-029-001) to S.J.B

\section{Author contributions}

M.H.L. conducted the field research. M.H.L. and C.P.L. conducted the spectral analyses. S.J.B. and I.M.T. wrote the main text. All authors contributed to the data analysis and reviewed the manuscript. 


\section{Additional information}

Supplementary information accompanies this paper at http://www.nature.com/ scientificreports

Competing financial interests: The authors declare no competing financial interests. How to cite this article: Liu, M.-H., Blamires, S.J., Liao, C.-P. \& Tso, I.-M. Evidence of bird dropping masquerading by a spider to avoid predators. Sci. Rep. 4, 5058; DOI:10.1038/ srep05058 (2014).
(1) This work is licensed under a Creative Commons Attribution-NonCommercialNoDerivs 3.0 Unported License. The images in this article are included in the article's Creative Commons license, unless indicated otherwise in the image credit; if the image is not included under the Creative Commons license, users will need to obtain permission from the license holder in order to reproduce the image. To view a copy of this license, visit http://creativecommons.org/licenses/by-nc-nd/3.0/ 